\title{
Medial Epicanthoplasty Using a Modified Skin Redraping Method
}

\section{Hong Lim Choi ${ }^{1}$, \\ Myung Chul Lee', Young Seok Kim², Dae Hyun Lew ${ }^{2}$}

${ }^{1} J W$ Jeong Won Plastic Surgery Clinic; ${ }^{2}$ Institute for Human Tissue Restoration, Department of Plastic and Reconstructive Surgery, Yonsei University Health System, Severance Hospital, Seoul, Korea

No potential conflict of interest relevant to this article was reported.
Background The presence of an epicanthal fold is a distinctive characteristic of the eyelids in Asian population. Various medial epicanthoplasty techniques have been introduced to achieve aesthetic appearance. The objective of this article is to describe our modification of the previously performed medial epicanthoplasty procedures, minimizing deterioration of the original ciliary position and maximizing natural appearance.

Methods From December of 2011 to February of 2013, total 386 patients with epicanthal folds have undergone medial epicanthoplasties using modified skin redraping method. The design of a new medial epicanthus, subciliary line of the lower eyelid and supratarsal fold line is crucial to this method. Intramuscular dissection and myotomy of the preseptal orbicularis oculi muscle are necessary to obtain natural eyelid position.

Results Most of the patients showed satisfactory results aesthetically during the follow up period; from 3 months to 12 months, a mean of 7.5 months postoperatively. There was no recurrence of the epicanthal fold. There was not any patient who complained about postoperative complications including ectropion and visible scars around the medial canthal area.

Conclusions The results indicate that medial epicanthoplasty using a modified skin redraping method is reliable and feasible procedure to correct epicanthal fold maintaining natural eyelid appearance.

Keywords Epicanthus, Fold, Correction, Natural eyelid

\section{INTRODUCTION}

The epicanthal fold is a skin fold over the lacrimal lake resulting from the certain anatomical structures including orbicularis oculi muscle, medial palpebral tendon and adjacent structures in the medial canthal area $[1,2]$. This fold is the medial extension of the upper eyelid skin and covers a portion of the lacrimal lake. The

Received: Jan 28, 2014 Revised: Feb 12, 2014 Accepted: Feb 13, 2014 Correspondence: Dae Hyun Lew Department of Plastic and Reconstructive Surgery, Yonsei University Health System, Severance Hospital, 250 Seongsanro, Seodaemun-gu, Seoul 120-752, Korea. E-mail: dhlew@yuhs.ac

Copyright @ 2014 The Korean Society for Aesthetic Plastic Surgery.

This is an Open Access article distributed under the terms of the Creative Commons Attribution Non-Commercial License (http://creativecommons.org/licenses/by-nc/3.0/) which permits unrestricted non-commercial use, distribution, and reproduction in any medium, provided the original work is properly cited. $\quad$ www.e-aaps.org eyes with epicanthal fold are usually unattractive aesthetically and cause some restrictions when performing double eyelidplasty and other ancillary procedures [3]. It has been investigated that the incidence of epicanthal fold is 50-90\% in Korean and Japanese populations [4]. Medial canthal area exhibits distinctive anatomic variations according to the presence or absence of the folds, and classified into four types (Table 1) [5].

Although an epicanthal fold is not a pathologic condition, numerous procedures have been introduced to correct this structure. Prominent epicanthal folds can be worsened when performing double eyelidplasty only without medial epicanthoplasty, because double eyelidplasty causes more tension on the epicanthal fold and results in an unpleasant round medial canthus [6]. Surgical techniques to improve epicanthal fold are mostly based on the concept of local flaps, besides they were developed and transformed to overcome drawbacks of previously conducted procedures. These tech- 
Table 1. Classification of medial epicanthal fold

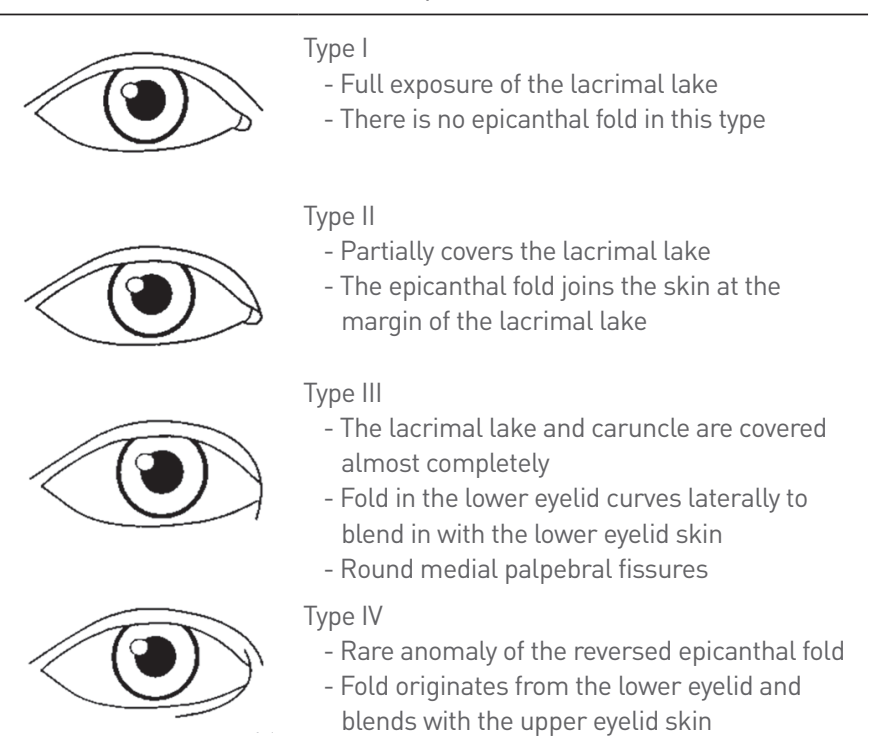

niques include the Mustarde four-flap [7], V-M plasty [8], V-W plasty (Uchida procedure) [9], modified V-Y flap [10], and various Z-epicanthoplasty techniques [11]. However, there are potential disadvantages with these techniques such as unnatural ciliary position of the lower eyelids, prominent scarring caused by excessive tension and limitations to obtain pretarsal fold running parallel to the upper eyelid margin. Medial epicanthoplasty using the skin redraping method was introduced to overcome previously mentioned problems [12]. Nevertheless, there were still disadvantages including medial canthal contracture due to superficial dissection and volume deficiency of the lower eyelid.

We propose a modification of the skin redraping method. There are supplementary points and procedures compared with the conventional technique, for instance, the designation of subciliary and supratarsal fold line, intramuscular dissection and preseptal orbicularis myotomy without muscle resection.

\section{METHODS}

Medial epicanthoplasties using modified skin redraping method were performed on 386 patients from December of 2011 to February of 2013. There were 43 male and 343 female patients. The average age was 24 years (range from 16 to 52 years). The followup period was 3 months to 12 months, with an average of 7.5 months. Patients were evaluated to identify the presence of entropion or ectropion, exophthalmos or enophthalmos, and a medical history of keloid or hypertrophic scar. All of the epicanthal folds in above group belonged to type II or III, according to the Park's classification (Table 1) [5]. 56 patients underwent only epicanthoplasty. 330 patients underwent simultaneous double eyelidplasty.

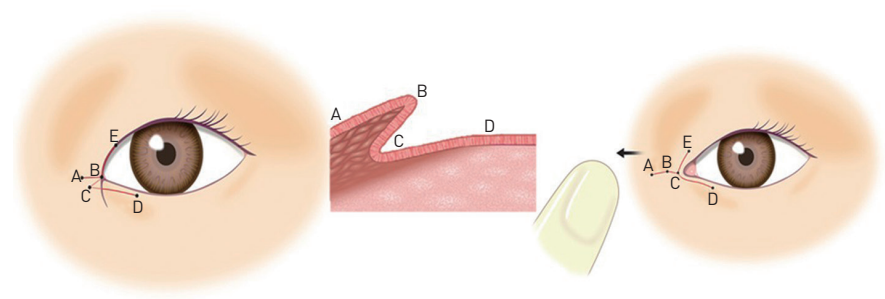

Fig. 1. Design. The incision line is designed by drawing "Y-shape" line passing through points $A$ to $C, C$ to $D$, and $C$ to $E$. Local anesthetic is infiltrated on the incision site, medial canthal region, and medial portion of the upper and lower eyelid.

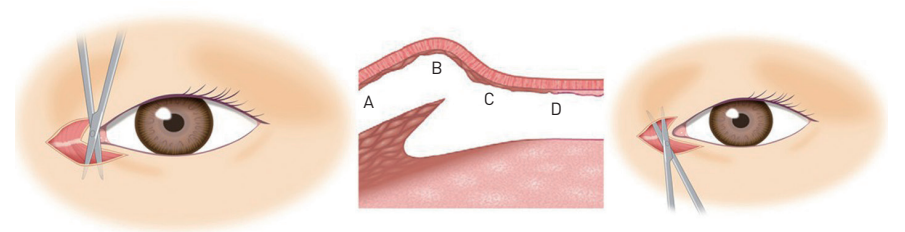

Fig. 2. Incision and dissection. Skin incisions are made from point $A$ to point $D$ and point $C$ to point $E$. The skin flap is elevated with intramuscular dissection by cutting the dense connective tissue between skin and orbicularis muscle of the lower and upper medial canthal area.

\section{Surgical technique}

The surgical procedures were performed under local anesthesia with the patients in supine position. Before the beginning of surgical designs, the amount of release was decided on the basis of two important factors, which were intercanthal distance and amount of lacrimal lake exposure. Point A was the desired location for the new medial epicanthus, and point $B$ was where the horizontal line passing point A met the edge of the original epicanthal fold (Fig. 1). After pulling the skin of the medial canthal area to the medial direction, point $\mathrm{C}$ was designated just medial to the lacrimal lake, which was similar to the symmetrical point of A on the basis of B. Point $\mathrm{D}$ was marked near the area where the subciliary line of the lower eyelid intersected the vertical line meeting the medial limbus. Point $\mathrm{E}$ was marked along with the supratarsal fold line. Point $\mathrm{D}$ and $\mathrm{E}$ were changeable according to the amount of movement and release of the medial canthus.

The incision line was designed by drawing "Y-shape" line passing through points A to C, C to D, and C to E. Local anesthetic was infiltrated on the incision site, medial canthal region, and medial portion of the upper and lower eyelid (Fig. 1). Skin incisions were made from point $\mathrm{A}$ to point $\mathrm{D}$ and point $\mathrm{C}$ to point $\mathrm{E}$. The skin flap was elevated with intramuscular dissection by cutting the dense connective tissue between skin and orbicularis muscle of the lower and upper medial canthal area (Fig. 2). The extent of dissection on skin flap and myotomy of the underlying orbicularis muscle should be graded depending on how much the epicanthal fold is to be released. Medial raphe was freed from upper preseptal 

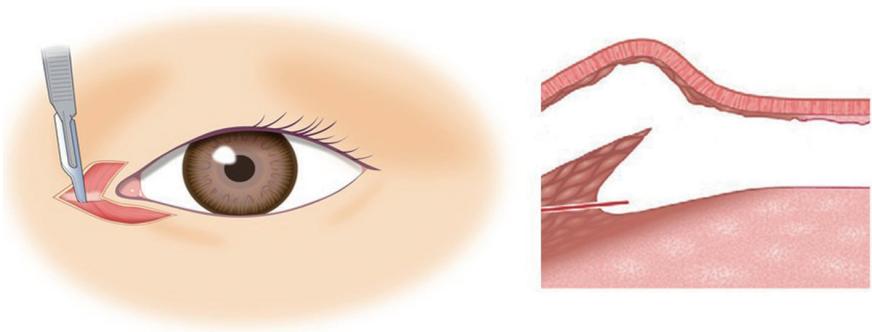

Fig. 3. Myotomy and release. The extent of the dissection of the skin flap and myotomy of the underlying orbicularis muscle should be graded depending on how much the epicanthal fold is to be released. Medial raphe is freed from upper preseptal muscle with bipolar coagulator. With this, most of the epicanthal fold and tension can be released. During dissection, there are two important points to be considered. First, for dissection plane of skin flap, intramuscular dissection is safer than raising subdermal flap which can cause postoperative contracture and pigmentation. Second, procedures manipulating the preseptal muscles should be just myotomy, not removal.
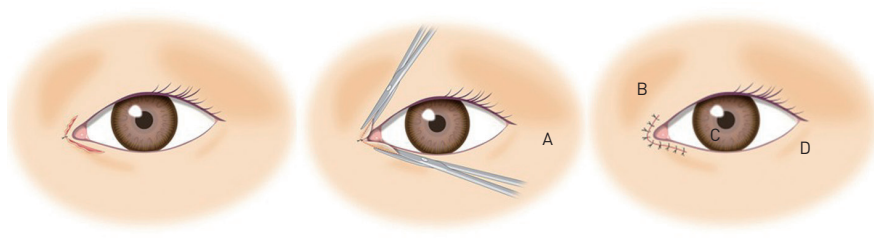

Fig. 4. Redrape and closure. Point $A$ was sutured to point $C$ on the medial canthus. At this time, point $\mathrm{C}$ of the skin flap is laid on the subciliary line for redraping of the skin flap. The excessive area of skin flap is trimmed and the subciliary incision was closed. The dogear on the supratarsal fold line is also removed and sutured. There are three points to be reminded during this process. First, key suture should be placed at the exact point of the new medial epicanthus. Second, excessive skin removal should be minimized. Third, skin closure has to be undergone without tension.

muscle with bipolar cautery. With this, most of the epicanthal fold and tension can be released. During dissection, there are two important points to be considered. First, for dissection plane of skin flap, intramuscular dissection is safer than raising subdermal flap which can cause postoperative contracture and pigmentation. Second, procedures manipulating the preseptal muscles should be just myotomy of medial raphe, not removal (Fig. 3). Point A was sutured to point $\mathrm{C}$ on the medial canthus. At this time, point $\mathrm{C}$ of the skin flap was laid on the subciliary line for redraping of the skin flap and additional traction of point $\mathrm{C}$ should avoided. The excessive area of skin flap was trimmed and the subciliary incision was closed. The dog-ear on the supratarsal fold line was also removed and sutured. There are three points to be reminded during this process. First, key suture should be placed at the exact point of the new medial epicanthus; repairing point $\mathrm{A}$, underlying orbicularis and point $\mathrm{C}$ sequentially with \#6-0 nylon sutures. Second, excessive skin removal should be minimized. Third, skin closure

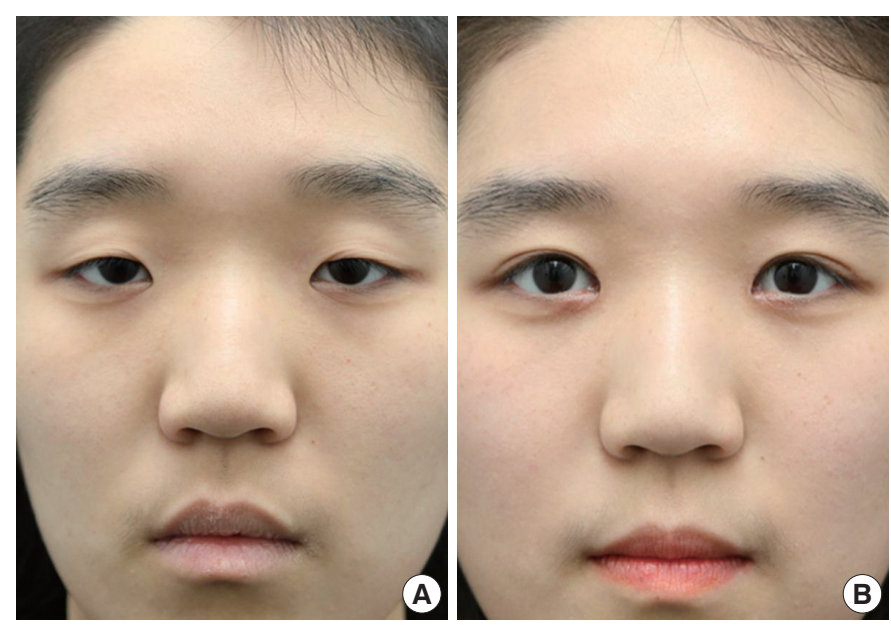

Fig. 5. (A) Preoperative view. (B) Postoperative 7 months after correction of the epicanthal fold and double eyelidplasty. Note the wider palpebral fissure, relaxed medial epicanthus and minimal scarring.
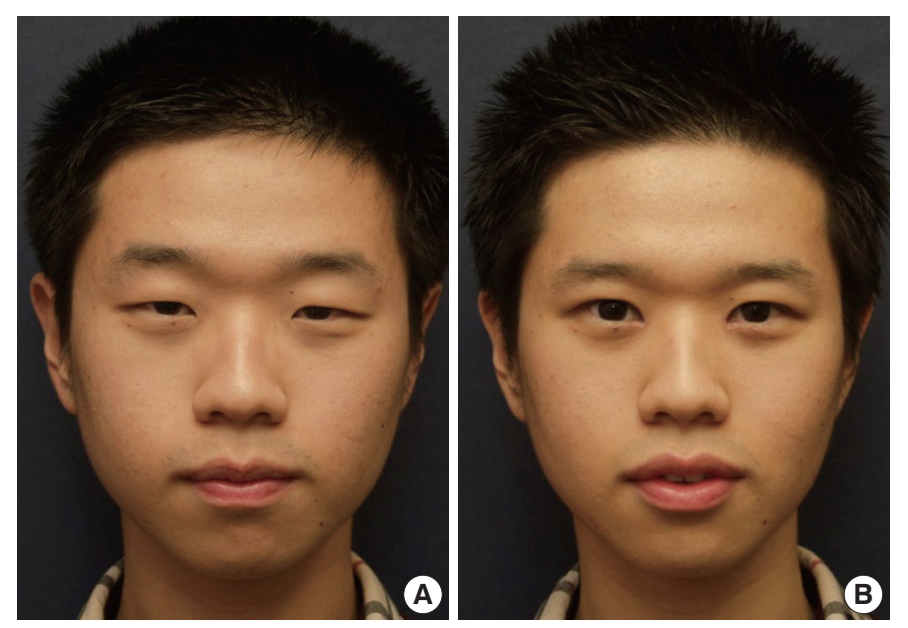

Fig. 6. (A) Preoperative view with ptosis. (B) Postoperative 9 months after correction of the epicanthal fold and levator plication procedure. Increased horizontal length of bilateral eyelids and vertical width of palpebral fissures were noted.

has to be undergone without tension, and additional sutures for repairing inner layer were not needed (Fig. 4).

\section{RESULTS}

A total of 386 patients underwent operative procedures employing the described surgical techniques; 330 of this group went through with simultaneous double eyelidplasty. Most of the patients obtained aesthetically satisfactory results being followed up from 3 months to 12 months postoperatively (Fig. 5, 6).

The mean follow-up period was 7.5 months. There was no recurrence of the epicanthal folds and wound related complications. Patients were followed up with interviews and photographs were 
taken at the same time. There was not any patient who complained about visible scarring at the medial epicanthal area. Tenderness and swelling in a small number of patients usually lasted up to 1-2 months and disappeared without revision or additional procedures.

\section{DISCUSSION}

The medial epicanthal fold is defined as a semilunar fold of skin that runs downward from the upper eyelid at the side of the nose with its concavity directed to the inner canthus. These epicanthal folds result in a round-looking inner eye and make the palpebral fissure narrower. It has been suggested that epicanthoplasty should be focused on several anatomic manifestations; the arrest in generation of the open epicanthus, the excessive development of skin on the root of the nose, a redundant attachment of orbicularis muscle and fibrofatty tissues beneath the fold, and abnormal forces of the orbicularis muscle resulting in tension on the skin [12].

The epicanthal fold, in addition to the eyelid with single fold, is a distinctive feature of East Asian populations, which are regarded as less attractive appearance. Epicanthoplasty can create a sharp medial corner of the epicanthus and widen the palpebral fissure which allows the eyes look more open. Many epicanthoplasty methods had been introduced to correct epicanthi fold, such as elliptical excision, Z-plasty [4-6], W-plasty [8], horizontal Y-V advancement [2] and other modified techniques [3,10,11]. Certain methods, however, created deficiency of the skin tissue and prominent scarring in the medial canthal area, which does not contribute to the attractive appearance.

Medial epicanthoplasty using the skin redraping method was established by Oh YW in 2007 [12]. This technique went after "tension free epicanthoplasty" because a prominent scar of the medial canthal area after epicanthoplasty could be caused by excessive tension and rigidity. Two independent procedures were performed at both muscle and skin tissues. First, preseptal orbicularis muscle was released. Second, tension on the skin was released by dissecting the adhesion between skin and orbicularis muscle. Nevertheless, there were several limitations including postoperative contracture and pigmentation due to superficial dissection on the subdermal plane, and downward direction of the cilia of the lower eyelid.

The authors' modifications were focused on those drawbacks of the conventional skin redraping method. First, intramuscular dissection is safer than raising subdermal flap superficially, which can cause postoperative contracture, hypertrophic scar, long lasting edema, and pigmentation. We can raise the lower flap in various surgical planes. Musculocutaneaous flap has limitation of moving, so may have some limitations in redraping, and can result in tension on the suture site consequently. On the contrast, pure skin only flap has good mobility, however has some limitations that we described above. Second, preseptal orbicularis muscles and medial raphe should be released by myotomy, not myectomy of muscular tissues for prevention of volume deficiency of lower lid. Third, removal of the minimal amount of remnant skin and dog ear tissue is recommended for reduction of tension. If adequately dissected, some remnant tissues are noted in lower lateral part. Trimming out is not recommended, but they should be sutured to medial side to augment and reduce tension in medial side. Fourth, Y-shape incision involving subciliary line of the lower eyelid and supratarsal fold line of the upper eyelid allows operator to mobilize the position of medial canthus horizontally and vertically. Appropriately designed supratarsal fold line can be extended to the double fold line for concomitant eyelidplasty. The other purpose of extending incision to upper eyelid is the prevention of dog ear deformity. With conventional redraping method, dog ear deformity can be easily met at the end of trimming site in upper lid. The lower part tissue should be preserved to maintain configuration and volume of lower cilia, but more active trimming the upper part is recommended to achieve 'deep set eyes'. Aforementioned modifications helped lower cilia to preserve their original position and direction avoiding downward distortion and even ectropion.

One of the most common and painful result is excessive vertical, horizontal enlargement and resulting caruncle exposure. The cause of this event is overestimation, over-excision of soft tissues and tension. In addition, excessive release should be avoided because it may result in too strong and sharp appearance.

Care must be taken to prevent complications. Medial epicanthus is close to the lacrimal canaliculi, and anatomical structures should be considered during the procedure. Temporary epiphora caused by partial obstruction of the lacrimal canaliculi from swelling can occur. These can be resolved without additional treatments. There are possibilities of certain degree of asymmetry.

\section{CONSLUSIONS}

Medial epicanthoplasty using a modified skin redraping method was performed to upgrade the conventional method. Authors' procedures including Y-shape incision, intramuscular dissection, releasing with myotomy of medial raphe and minimal skin resection helped preserving original ciliary position and maximizing natural appearance. As a conclusion, medial epicanthoplasty using a modified redraping method is very effective method to correct medial epicanthal fold.

\section{REFERENCES}

1. Yamamoto H, Morikawa K, Uchinuma E, et al. An anatomical study of the medial canthus using a three-dimensional model. Aesthetic Plast Surg 2001;25:189-93.

2. Li FC, Ma LH. Double eyelid blepharoplasty incorporating epicanthoplasty using Y-V advancement procedure. J Plast Reconstr Aesthet 


\section{Surg 2008;61:901-5.}

3. Cho BC, Lee KY. Medial epicanthoplasty combined with plication of the medial canthal tendon in Asian eyelids. Plast Reconstr Surg 2002; 110:293-300.

4. Park JI. Modified Z-epicanthoplasty in the Asian eyelid. Arch Facial Plast Surg 2000;2:43-7.

5. Park JI. Z-epicanthoplasty in Asian eyelids. Plast Reconstr Surg 1996; 98:602-9.

6. Park JI, Park MS. Park Z-epicanthoplasty. Facial Plast Surg Clin North Am 2007;15:343-52.

7. Mustarde JC. Epicanthus and telecanthus. Int Ophthalmol Clin 1964;4:
$359-76$.

8. Lin SD. Correction of the epicanthal fold using the VM-plasty. Br J Plast Surg 2000;53:95-9.

9. Uchida J. A surgical procedure for blepharoptosis vera and for pseudoblepharoptosis orientalis. Br J Plast Surg 1962;15:271-6.

10. Kao YS, Lin CH, Fang RH. Epicanthoplasty with modified Y-V advancement procedure. Plast Reconstr Surg 1998;102:1835-41.

11. Yoo WM, Park SH, Kwag DR. Root z-epicanthoplasty inAsian eyelids. Plast Reconstr Surg 2002;109:2067-71.

12. Oh YW, Seul CH, Yoo WM. Medial epicanthoplasty using the skin redraping method. Plast Reconstr Surg 2007;119:703-10. 